\title{
Inhibition of Ornithine Carbamoyltransferase by Arginase among Yeasts: Correlation with Energy Production, Subcellular Localization and Enzyme Synthesis
}

\author{
By STEPHAN VISSERS, * LUIS ANTONIO URRESTARAZU, \\ JEAN-CLAUDE JAUNIAUX AND JEAN-MARIE WIAME \\ Laboratoire de Microbiologie, Faculté des Sciences, Université Libre de Bruxelles, and \\ Institut de Recherches du C.E.R.I.A., 1 Av. E. Gryson, B-1070 Brussels, Belgium
}

(Received 25 February 1981; revised 14 August 1981)

\begin{abstract}
Thirty-two yeast species belonging to twelve genera were examined for the occurrence of inhibition of ornithine carbamoyltransferase by arginase (epiarginasic regulation) and related properties. Obligate aerobes were devoid of this regulation. Among fermenting species, Schizosaccharomyces and budding genera had different properties: all Schizosaccharomyces species were devoid of this regulation whereas all species of budding yeasts showing a weak or absent Pasteur effect had this regulation. Strains showing a strong Pasteur effect and taxonomically related to Saccharomyces (Kluyveromyces) had the regulation, whereas species classified in genera that include species which are obligate aerobes did not.

We confirm that the absence of epiarginasic regulation is correlated with a mitochondrial localization of ornithine carbamoyltransferase but not with the function of the mitochondria in Schizosaccharomyces japonicus. In this case, compartmentation could serve to channel anabolic and catabolic functions. Although, in general, the arginase of 'negative epiarginasic' species had no affinity for cytosolic ornithine carbamoyltransferase, one exception was found (Hansenula anomala).

Regulation by repression of ornithine carbamoyltransferase and induction of arginase by arginine was the general rule. These types of regulation of enzyme synthesis were only absent in yeasts in which compartmentation is present and could serve as the basis for anabolism-catabolism exclusion.
\end{abstract}

\section{INTRODUCTION}

Saccharomyces cerevisiae has a peculiar regulatory mechanism which excludes interference between biosynthesis and degradation of arginine. Arginase, the first enzyme of the arginine degradative pathway, converts this amino acid into ornithine and urea. This enzyme is able to inhibit ornithine carbamoyltransferase (OTCase), the biosynthetic enzyme which converts ornithine plus carbamoylphosphate into citrulline and phosphate. This inhibition of OTCase was first observed 'in situ' in cells which were permeabilized, leaving macromolecules inside the cells (Béchet $\&$ Wiame, 1965). The nature of the inhibitory molecule was established in cell-free extracts and was shown to be arginase. The binding of the two enzymes is reversible and is affected by arginine and ornithine (Messenguy \& Wiame, 1969). The ornithine receptor site is on OTCase and is distinct from the catalytic site. Ornithine at a higher concentration $(20 \mathrm{~mm})$ than the $K_{\mathrm{m}}$ value for catalysis $(3 \mathrm{~mm})$ provokes a limited inhibition on its own. Arginine acts together with arginase, but alone does not inhibit OTCase. Arginase maintains full catalytic activity when complexed with and inhibiting OTCase (Messenguy et al., 1971; Penninckx, 1975; Penninckx \& Wiame, 1976). This system 
of regulation, termed 'epiarginasic regulation', is not present in all yeasts (Wiame, 1971), but it has been reported in Bacillus subtilis (Issaly \& Issaly, 1974).

The OTCases of Neurospora crassa and liver are mitochondrial enzymes and thus are not accessible to cytosolic arginase (Weiss \& Davis, 1973; Gamble \& Lehninger, 1973), making improbable any regulation by association of these two enzymes. However, in Sacch. cerevisiae both enzymes are outside the mitochondria (Urrestarazu et al., 1977) and are very likely cytosolic, thus affording the opportunity for regulation by interaction.

The main purpose of the work described in this paper was to try to evaluate the evolutionary process which led to this specific and original regulatory process. The fact that ornithine belongs to both the biosynthetic and degradative pathways creates the possibility of a futile cycle resulting from resynthesis of arginine from ornithine. This would lead to the loss of at least five energy-rich bonds (one for the synthesis of glutamine, two for the synthesis of carbamoylphosphate and two for the synthesis of argininosuccinate). An additional energy-rich bond is necessary to recover ammonia from urea (Roon et al., 1972). The large expenditure of energy in futile resynthesis of arginine would probably be more damaging than the loss of one $\mathrm{CO}_{2}$ per cycle. The epiarginasic regulation might be a way to avoid this waste. Our attention was then focused on the peculiarity of Sacch. cerevisiae in obtaining its energy from an energy-poor process (fermentation) which is retained even in the presence of oxygen when sugar is the energy source (Ephrussi et al., 1956). In this organism, perhaps ATP limitation was an important factor in the original selection of the epiarginasic regulation.

\section{METHODS}

Strains. The yeast strains used are listed in Table 1. They belong to the Endomycetales and the Fungi Imperfecti (Lodder, 1970) and came from our own collection (IRC) or from the Centraalbureau voor Schimmelcultures, The Netherlands (CBS). The diploid strain of Hansenula anomala was obtained by crossing NRRL Y-366-8 with NRRL Y-2153-4 (Northern Regional Research Laboratory, Ill., U.S.A.).

Media. Yeasts were maintained on complex medium solidified with $2 \%(\mathrm{w} / \mathrm{v})$ agar (Ramos \& Wiame, 1979). Analysis of growth and cell production were performed in medium $\mathbf{M}$, composed of a mineral solution to which separate sterile glucose and sterile vitamin solutions were added. The mineral and vitamin solutions were those previously described by Grenson et al. (1966) for medium no. 150. The nitrogen sources used (as indicated in the text) were $0.01 \mathrm{M}$-ammonium sulphate and/or $1 \mathrm{mg}$ arginine $\mathrm{ml}^{-1}$. The preparation of the mineral solution was slightly modified in later experiments (with Schizosaccharomyces japonicus var. versatilis) by separate sterilization of the heavy metals and trace elements (Ramos \& Wiame, 1979). For Schiz. octosporus, 0.1\% (w/v) yeast extract was added to the minimal medium. For anaerobic cultures the media were supplemented with $0.1 \mathrm{ml}$ Tween 80 and $1 \mathrm{mg}$ ergosterol $\left(1 \mathrm{mg} \mathrm{ml}{ }^{-1}\right.$ in methanol) per $100 \mathrm{ml}$ medium.

Growth conditions. All the cultures were maintained at $29^{\circ} \mathrm{C}$. Liquid cultures were grown on a rotary shaker in flasks with provision for turbidity estimation. Anaerobiosis was obtained in liquid cultures by two successive evacuations of gas and flushing with nitrogen. Using such conditions, strict aerophiles like Cryptococcus neoformans did not grow but microaerophiles like Schiz. pombe did. To determine the differential rate of synthesis of an enzyme or of ethanol production, several flasks of the same culture were followed in parallel and harvested at different turbidities. Two qualitative tests of ability to grow anaerobically on solid media were also performed. In the first, cotton plugs were inserted partway into test tubes containing agar $(2 \%, \mathrm{w} / \mathrm{v})$ stabs of the organism. Pyrogallol crystals $(0.5 \mathrm{~g})$ and 4 drops of $5 \mathrm{M}-\mathrm{KOH}$ were placed on top of the cotton plug, and the test tube was closed with a rubber stopper. The second test used an apparatus applicable to both test tubes and Petri dishes, as described by Brewer \& Allgeier (1966) and available commercially (BBL) under the name of GasPak; the tubes were examined after $10 \mathrm{~d}$. For the epiarginasic inhibition test in situ, the cells were transferred from one medium to another by filtration of the cultures on Millipore filters, except for $\mathrm{Cr}$. neoformans which had to be centrifuged because it warped the filters. The growth in liquid medium was followed by measurement of turbidity at $660 \mathrm{~nm}$. An $A_{660}$ of 1 in the Beckman B spectrophotometer or of 3.4 in the Gilford 2000 spectrophotometer corresponded to $0.53 \mathrm{mg}$ protein ( $\mathrm{ml}$ culture) ) $^{-1}$ as determined with Sacch. cerevisiae.

Cell extracts and cell permeabilization. Samples from cultures in the exponential phase of growth were filtered on Millipore filters or centrifuged in the presence of cycloheximide $\left(2 \mu \mathrm{g} \mathrm{m}^{-1}\right)$ and washed with cold water. The pellets were stored in ice and subsequently resuspended in the buffer appropriate to the enzyme assay. Cells were broken in a French press precooled to $4^{\circ} \mathrm{C}$, and cell debris was removed by centrifugation at $22000 \mathrm{~g}$ for $20 \mathrm{~min}$. The crude extract was desalted on a Sephadex G-25 fine column (Pharmacia) preconditioned and eluted with the buffer appropriate to the enzyme assay. 
Table 1. Name and origin of the species (according to Lodder, 1970)

\author{
Species \\ Brettanomyces lambicus Kufferath \& van Laer \\ Candida tropicalis (Cast.) Berkhout \\ Candida utilis (Henneberg) Lodder \& van Rij ex Torulopsis utilis \\ Cryptococcus neoformans (Sanfelice) Vuillemin \\ Debaryomyces hansenii (Zopf) Lodder \& van Rij \\ Debaryomyces hansenii ex Debaryomyces kloeckeri \\ Debaryomyces hansenii ex Debaryomyces subglobosus \\ Debaryomyces hansenii ex Debaryomyces nicotianae \\ Debaryomyces marama di Menna \\ Debaryomyces phaffii Capriotti \\ Hanseniaspora valbyensis Klöcker \\ Hansenula anomala (Hansen) H. \& P. Sydow \\ Hansenula anomala var. anomala \\ Hansenula wingei Wickerham \\ Kluyveromyces fragilis (Jörgensen) van der Walt ex Sacch. fragilis \\ Kluyveromyces lactis (Dombrowski) van der Walt \\ Pichia dispora (Dekker) van Rij \\ Pichia fermentans Lodder \\ Pichia fluxuum (Pfaff \& Knapp) van Rij \\ Saccharomyces cerevisiae Hansen \\ Saccharomyces fermentati (Saito) Lodder \& van Rij \\ Saccharomyces fermentati ex Debaryomyces dekkeri \\ Saccharomyces kloeckerianus van der Walt ex Deb. globosus \\ Saccharomycodes ludwigii Hansen \\ Schizosaccharomyces japonicus Yukawa \& Maki var. japonicus \\ Schizosaccharomyces japonicus Yukawa \& Maki var. versatilis \\ (Wickerham \& Duprat) Sloof \\ Schizosaccharomyces malidevorans Rankine \& Fornachon \\ Schizosaccharomyces octosporus Beijerinck \\ Schizosaccharomyces octosporus \\ Schizosaccharomyces pombe Lindler \\ Torulopsis candida (Saito) Lodder \\ Torulopsis colliculosa (Hartmann) Saccardo
}

Origin

IRC L-1-3
IRC L-7-4
IRC L-7-14
CBS 132
CBS 767
CBS 770
CBS 792
CBS 811
CBS 1958
CBS 4346
IRC L-4-1
IRC L-5-6
NRRL Y-366-8 $\times$ NRRL Y-2153-4
IRC L-5-19
IRC L-3-6
CBS 683
CBS 794
IRC L-5-16
CBS 2287
IRC $\sum 1278 b$
CBS 818
CBS 813
CBS 764
IRC L-4-3
CBS 354
CBS 103

CBS 5.557

IRC L-0-6

CBS 371

IRC L-0-8

CBS 940

CBS 133

Permeabilization of fresh cells was achieved by treatment with nystatin (Labaz, Brussels, Belgium) as described by Bechet \& Wiame (1965) in Tris/HCl buffer $(0 \cdot 1 \mathrm{M}, \mathrm{pH} 8)$. Permeabilization of Schiz. japonicus strains required a more acid buffer (sodium acetate, $0.05 \mathrm{M}, \mathrm{pH} \mathrm{5)}$. In contrast, all the yeasts could be permeabilized with $0.4 \%(w / v)$ Triton X-100 (Serva, Heidelberg, Germany) in Tris/HCl buffer $(0.1 \mathrm{M}, \mathrm{pH} 8)$. All the permeabilization treatments were performed at $30^{\circ} \mathrm{C}$ for $40 \mathrm{~min}$ with shaking.

For in vitro cross-inhibition tests between OTCase and arginase of different origins and the two enzymes of Sacch. cerevisiae, all extracts were desalted. Cells cultured on ammonia medium provided OTCase, whereas they were poor in arginase. Cells cultured on arginine medium provided arginase. In the case of Sacch. cerevisiae, desalted crude extract was used, whereas for the other species, OTCase was eliminated by the following procedure. Nucleic acids were precipitated by adding protamine sulphate (14-28\% of protein weight). The supernatant was desalted on Sephadex G-25 fine preconditioned with sodium maleate buffer $(0.001 \mathrm{M}, \mathrm{pH} 6 \cdot 5)$. A sample containing 6000-37000 units of arginase ( $\left.\mu \mathrm{mol} \mathrm{h}{ }^{\prime}\right)$ was applied to an arginyl-Sepharose column as described by Penninckx et al. (1974) and eluted with an arginine gradient $(0-0.1 \mathrm{M})$. All operations were performed at $4{ }^{\circ} \mathrm{C}$.

Enzyme assays. Ornithine carbamoyltransferase (OTCase; EC 2.1.3.3) was measured at $30^{\circ} \mathrm{C}$ as described in Messenguy et al. (1971) except that $\mathrm{MgCl}_{2}$ was omitted. Tris/ $\mathrm{HCl}$ buffer $(0 \cdot 1 \mathrm{M}, \mathrm{pH} 8)$ was used for the determination of specific activities and in situ tests. For the determination of inhibition by excess ornithine, the enzyme activity was measured at $15^{\circ} \mathrm{C}$ in Tris/ $\mathrm{HCl}$ buffer $(0.1 \mathrm{M}, \mathrm{pH} 8.6)$. In vitro inhibition tests were done at $15^{\circ} \mathrm{C}$ in Tris $/ \mathrm{HCl}$ buffer $(0.1 \mathrm{M}, \mathrm{pH} 8)$, with $0.005 \mathrm{M}$-arginine and $0.06 \mathrm{M}$-ornithine as effectors. Arginase (EC 3.5.3.1) was assayed as described by Messenguy et al. (1971) and glucose-6-phosphate dehydrogenase (EC 1.1.1.49) and citrate synthase (EC 4.1.3.7) as described by Urrestarazu et al. (1977). Protein concentrations were estimated by the Lowry method with bovine serum albumin as a standard. except that the protein concentrations of chromatography column fractions were estimated by their absorbance at $280 \mathrm{~nm}$. Ethanol was measured as described in Bonnichsen (1963).

Enzyme subcellular localization. We used the technique of stepwise homogenization of spheroplast lysates described by Ryan et al. (1973). For this purpose Schiz. japonicus var. versatilis was grown aerobically in 
ammonia medium supplemented with $1 \%(\mathrm{w} / \mathrm{v})$ yeast extract, $0.001 \%(\mathrm{w} / \mathrm{v})$ ergosterol and $0 \cdot 1 \mathrm{M}-\mathrm{Tween} 80$. An isotonic mannitol solution $(1.8 \mathrm{M})$ was added to $25 \mathrm{~g}$ fresh yeast (harvested in the exponential phase of growth) to a volume of $35 \mathrm{ml}$ in the presence of $174 \mathrm{mg}$ Zymolase 5000 (Kirin Brewery, Tokyo, Japan) and 4 ml Helicase (I.B.F., Gennevilliers, France). Incubation was at $30^{\circ} \mathrm{C}$ for $120 \mathrm{~min}$ with continuous shaking. Spheroplasts were then separated by centrifugation in several aliquots and the pellets were processed separately as indicated in the legend to Fig. 6 (for details, see Urrestarazu et al., 1977). Glucose-6-phosphate dehydrogenase and citrate synthase were used as cytosolic and mitochondrial markers, respectively.

\section{RESULTS AND DISCUSSION}

\section{Epiarginasic regulation in situ}

Epiarginasic regulation was initially discovered by the abolition of OTCase activity when arginine was added to cells growing with ammonia as the sole nitrogen nutrient, OTCase activity being measured in nystatin-permeabilized cells (Béchet \& Wiame, 1965). However, further investigations, including the present study, have disclosed two main pitfalls.

First, all yeasts were able to synthesize arginase, which in virtually all cases was induced to a higher level by growth on arginine, but they reacted differently when arginine and ammonia were present in the medium. The main variability lay in their sensitivity to the ammonia effect, even in strains of Sacch. cerevisiae which had the potentiality to develop epiarginasic regulation. The presence of ammonia may inhibit arginase synthesis either directly or by inhibiting transport of arginine. A typical ammonia-insensitive strain is strain $1705 \mathrm{~d}$ in which the phenomenon was discovered; with our usual laboratory strain, $\Sigma 1278 \mathrm{~b}(\alpha)$, the ammonia effect was strong and the in situ epiarginasic reaction was weak (Dubois et al., 1974; Rytka, 1975). Both effects of ammonia may be avoided by shifting cells from ammonia to arginine as the only nitrogen nutrient.

The second pitfall resulted from a difference in permeabilization by nystatin. Some species belonging to genera other than Saccharomyces were rather insensitive to nystatin. Triton $\mathrm{X}-100$ could be used in such circumstances; this was the case for Schizosaccharomyces species. An unexpected but rare difficulty occurred with Schiz. japonicus. In this yeast, addition of arginine to the medium reduced the capacity of the cells to be permeabilized. This capacity was also influenced by aeration, which had other unfavourable effects on this yeast (Wickerham \& Duprat, 1945; Bulder, 1963). The lack of permeabilization could be detected by the absence of any enzyme activities after treatment of the cells, as well as by the absence of any leakage of internal amino acids.

Unless stated otherwise, all results (Table 2) are expressed as differential rates, i.e. activity per $\mathrm{ml}$ culture versus mass per $\mathrm{ml}$ culture, in cultures growing exponentially (Monod et al., 1952).

All species tested belonging to the genus Saccharomyces (Lodder, 1970) had a strong positive reaction (inhibition of OTCase activity by arginine). The species tested were Sacch. bayanus, Sacch. bayanus ex oviformis, Sacch. bayanus ex pastorianus, Sacch. cerevisiae ex turbidans, Sacch. chevalieri, Sacch. delbrueckii, Sacch. diastaticus, Sacch. fermentati, Sacch. kloeckerianus, Sacch. italicus and Sacch. rosei (Wiame, 1971, 1975). Only Sacch. cerevisiae is considered further. Apart from the Saccharomyces species, Saccharomycodes ludwigii, Torulopsis colliculosa, Kluyveromyces fragilis, $K$. lactis and Hanseniaspora valbyensis (Fig. 1a) gave a positive result in situ.

Typical negative results were obtained for many yeasts such as Candida utilis (Fig. $1 b$ ) and Schiz. pombe. Negative results could not be attributed to lack of arginase synthesis (see Table 3): although positive-reacting species were always arginase-rich, some very arginase-rich cells were negative, e.g. Torulopsis candida.

\section{Epiarginasic regulation in vitro}

A more direct but more laborious test for epiarginasic regulation can be performed by mixing the enzymes (Messenguy \& Wiame, 1969). Such in vitro tests allowed investigation of 
Table 2. Fermentation capacity in aerobic and in anaerobic conditions of growth, epiarginasic regulation and catalytic properties of the OTCases in different yeast species

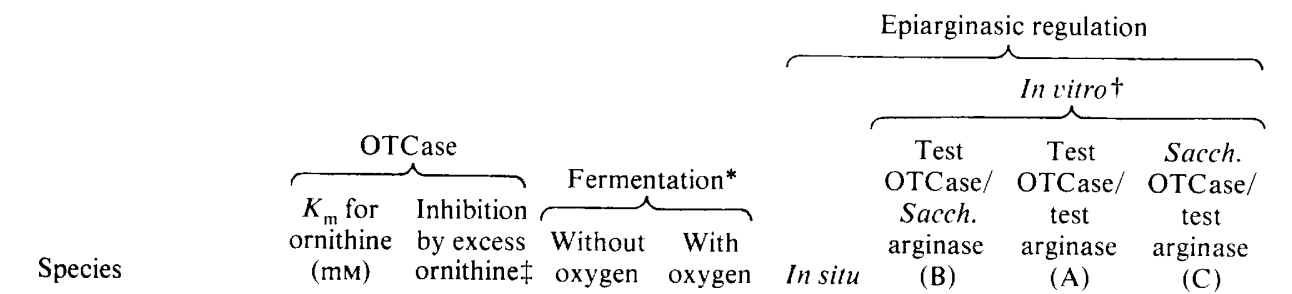

\section{OBLIGATE AEROBES}

\section{Cr. neoformans}

Deb. hansenii 767

Deb. hansenii 770

Deb. hansenii 792

Deb. hansenii 811

Deb marama

H. wingei

P. fluxuum

T. candida

In situ

(B)

(A)

(C)

\section{FACULTATIVE ANAEROBES WITH STRONG PASTEUR EFFECT}

C. tropicalis
C. utilis
Deb. phaffii
H'spora valbyensis
H. anomala L-5-6
H. anomala diploid
K. fragilis
K. lactis
P. fermentans
P. dispora

1
1
$3 \cdot 6$
$2 \cdot 2$
$3 \cdot 3$
$3 \cdot 7$
$0 \cdot 7$
$0 \cdot 6$
$0 \cdot 7$
$3 \cdot 8$

$\begin{array}{cc}+ & 27 \\ + & 23 \\ - & 58 \\ ++ & 42 \\ \text { trace } & 28 \\ \text { trace } & 19 \\ ++ & 27 \\ + & 27 \\ \text { weak } & 27 \\ + & 51\end{array}$

\begin{tabular}{l}
\multicolumn{1}{c}{0} \\
1 \\
$0 \cdot 5$ \\
$0 \cdot 5$ \\
0 \\
4 \\
$0 \cdot 3$ \\
2 \\
$0 \cdot 1$ \\
7
\end{tabular}

-
-
-
+
-
-
+
+
-
-

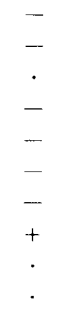

$\begin{array}{cc}- & - \\ - & \text { weak } \\ \text { weak } & - \\ - & - \\ - & + \\ + & + \\ + & + \\ . & + \\ . & \cdot\end{array}$

\section{FACULTATIVE ANAEROBES WITH WEAK PASTEUR EFFECT}

\section{Br. lambicus}

Schiz. japonicus var. jap.

Schiz. japonicus var. vers.

Schiz. malidevorans

Schiz. octosporus L-0-6॥

Schiz. octosporus 3711

Schiz. pombe

Sacch. cerevisiae

Sacch. fermentati 818

Sacch.fermentati 813

Sacch. kloeckerianus

S'codes ludwigii

T. colliculosa

$\begin{array}{cccc}0.7 & \text { weak } & 15 & 12 \\ 5.4 & + & 69 & 75 \\ 6.2 & + & 60 & 74 \\ 7.7 & \text { weak } & 52 & 46 \\ . & . & . & . \\ . & . & . & . \\ 1.6 & \text { weak } & 42 & 31 \\ 3 & + & 47 & 34 \\ 4.5 & + & 33 & 13 \\ 1.6 & + & . & . \\ 3.3 & + & 33 & 9 \\ 1.3 & ++ & 68 & 20 \\ 8.3 & + & 51 & 22\end{array}$

* Expressed as the ethanol concentration excreted into the medium when the cultures had reached an $A_{660}$ of 0.5 measured with the Beckman B spectrophotometer, corresponding to $0.265 \mathrm{mg}^{2}$ protein $\mathrm{ml}^{-1}$ with $S a c c h$. cerevisiae as reference.

† 'Test' denotes OTCase or arginase of the yeast under test; 'Sacch.' denotes OTCase or arginase of Sacch. cerevisiae. A, B and C correspond to the interactions shown in Fig. 2.

$\ddagger+$, Inhibition comparable to Sacch. cerevisiae; ++ , inhibition more intense than Sacch. cerevisiae; weak, inhibition present for ornithine at concentrations $\geqslant 10 \mathrm{~mm}$.

$\S$ See text for a description of the exceptional behaviour of these yeasts in the in situ assay.

II Growth of Schiz. octosporus was very poor on yeast extract medium under anaerobic conditions. In mineral medium there was no growth aerobically or anaerobically; thus the placing of this yeast among the facultative anaerobes is tentative.

I Other species of Saccharomyces have been considered previously (see text). All showed epiarginasic regulation. 


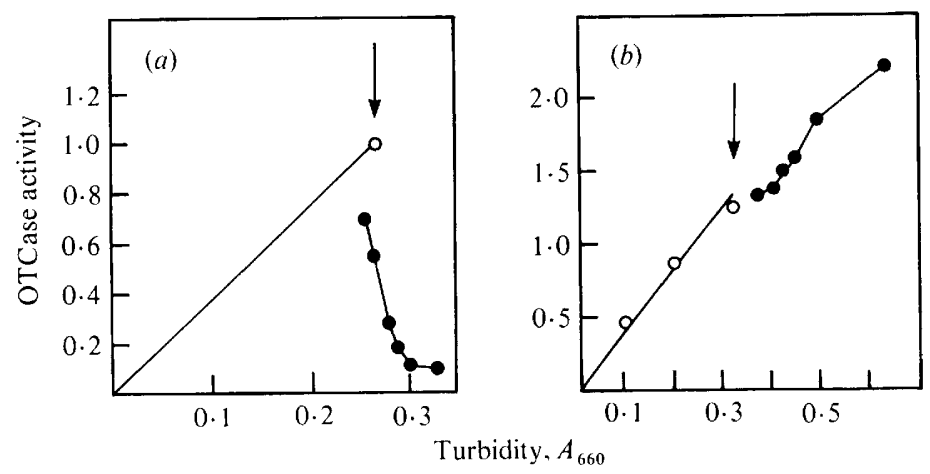

Fig. 1. In situ inhibition of OTCase from (a) Hanseniaspora valbyensis and (b) Candida utilis. The arrow indicates the stage of growth at which cells were shifted from ammonia $(O)$ to arginine $(O)$ medium. Samples of cells were permeabilized with nystatin. Activity is expressed in $\mu$ mol citrulline formed $\mathrm{h}^{-1}$ (ml culture $)^{-1}$. Turbidity was measured with a Beckman B spectrophotometer.

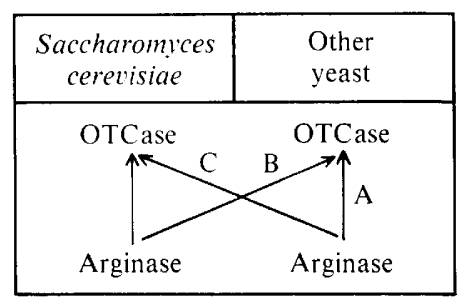

Fig. 2. Designation of cross-reactions between OTCase and arginase of different yeasts and the enzymes of Sacch. cerevisiae.

intergeneric or interspecies interactions between the two enzymes (cross-reaction). A partial purification was carried out to separate arginase from contaminating OTCase (see Methods). In the present work cross-reactions were limited to different yeasts in combination with Sacch. cerevisiae (Fig. 2). The following categories of results can be defined.

(1) Full positive couples. Interactions A, B and C (Fig. 2) occurred, i.e. arginase and OTCase of the tested yeast both exchanged with the Sacch. cerevisiae enzymes without qualitative modifications. This was the case for all the Saccharomyces species tested. It was also the case for most other yeasts giving a positive result in situ (Table 2). Apart from the Saccharomyces species, it occurred in K. lactis (Fig. 3), S'codes ludwigii and T. colliculosa.

(2) Full negative couples. Interactions A, B and C did not occur. This was the case for most yeasts giving negative results in situ. Examples were Deb. hansenii, T. candida, $C$. tropicalis, Br. lambicus, Schiz. pombe (Fig. 3) and Schiz.japonicus var. versatilis.

(3) Positive specific. Cross-reactions did not occur (B and $\mathrm{C}$ did not occur). The only example found was H'spora valbyensis (Fig. $1 a$, in situ; and Fig. 3, in vitro). In spite of a weak interaction in vitro, the test in situ was clearly positive.

(4) Positive semi-specific. (4-1) Interaction B did not occur. For example, K. fragilis OTCase was insensitive to Sacch. cerevisiae arginase, in contrast to the fully positive $K$. lactis. (4-2) No case of interaction $\mathrm{C}$ not occurring was found when interaction A occurred.

(5) Negative for its own enzyme(s) but positive for Sacch. cerevisiae enzyme(s). Interaction A did not occur but either interaction B or C did occur. (5-1) Hansenula anomala OTCase was insensitive to its own arginase as well as to that of Sacch. cerevisiae, but the arginase of $H$. anomala strongly inhibited Sacch. cerevisiae OTCase (Fig. 3). Such a reaction did not occur in $H$. wingei, an obligate aerobe. Note that $H$. anomala OTCase is mitochondrial and 

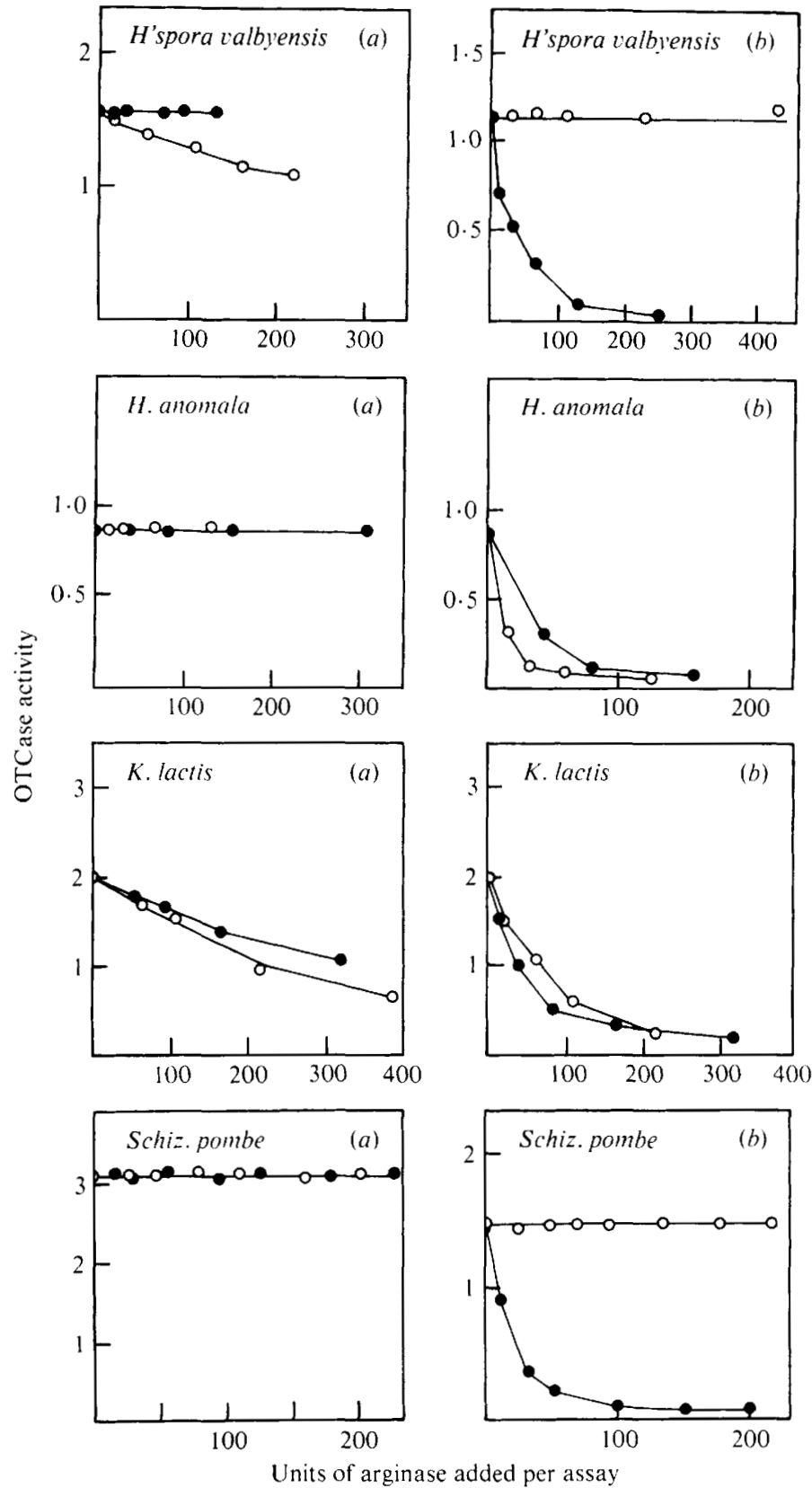

Fig. 3. Representative in vitro determinations of inhibition of OTCase from different yeasts: cross-reactions between a given yeast OTCase $(a)$ or Sacch. cerevisiae OTCase $(b)$ and the arginases from the yeast tested $(O)$ and from Sacch. cerevisiae (O). Enzyme activities are expressed in $\mu \mathrm{mol}$ product formed $h^{-1}$ per assay.

would thus be unable to react with its cytosolic arginase (Urrestarazu et al., 1977). (5-2) No case of an OTCase insensitive to its own arginase but sensitive to Sacch. cerevisiae arginase was found.

As proposed by Price et al. (1978), epiarginasic regulation may be a useful tool for the study of relationships between yeasts. However, too few cases have been analysed to establish 


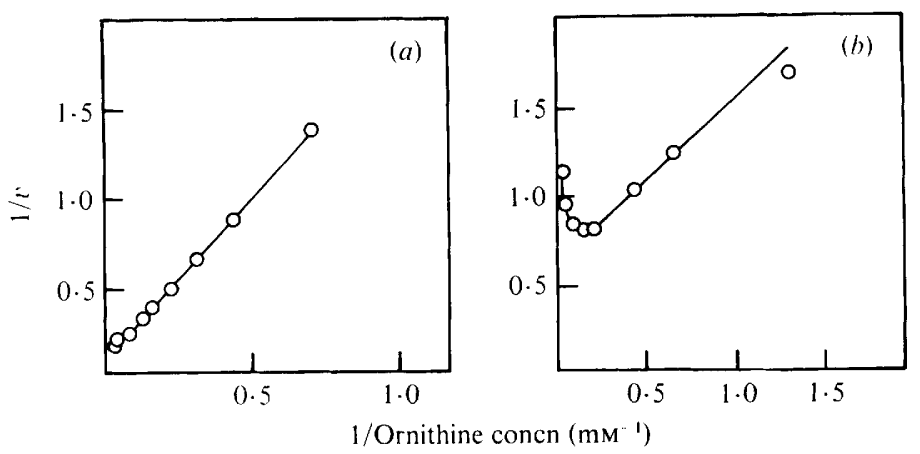

Fig. 4. Double reciprocal plots for ornithine saturation of ornithine carbamoyltransferase in Tris/ $\mathrm{HCl}$ buffer $(0.1 \mathrm{M}, \mathrm{pH} 8.6)$ at $15^{\circ} \mathrm{C}$ for $(a)$ Debaryomyces marama and (b) Cryptococcus neoformans.

a definite rule. Usually the species belonging to the same genus interacted similarly, except for $K$. lactis and $K$. fragilis. In other cases, differences paralleled strong biochemical distinctions. The exception of the two Torulopsis species is probably an apparent one. Indeed, these yeasts are Fungi Imperfecti and Van Uden \& Vidal-Luria (in Lodder, 1970) consider T. colliculosa (a full positive species) as the imperfect form of Sacch. fermentati (a full positive species), while $T$. candida (a full negative species) is considered as the imperfect form of Deb. hansenii or Deb. marama (both full negative species). The present study would favour the separation of the two Torulopsis species. The difference observed between $H$. wingei and $H$. anomala is worthy of some additional consideration, justified by their additional difference in fermentative capacity. Yeasts which were neither fully positive nor fully negative are the most promising for the refinement of phylogeny or for investigating the fine molecular structure involved in epiarginasic regulation; this is the case for Kluyveromyces, Hansenula and Hanseniaspora. Among these, $H$. anomala (class 5-1) is of particular interest. Its arginase did not react with its own OTCase, which is mitochondrial, but it did react with the cytosolic OTCase of a distantly related organism. This raises the possibility that the difference between the two OTCases is linked with the capacity of OTCase to migrate into the mitochondria.

\section{OTCase interaction with L-ornithine: $K_{m}$ values and inhibition}

Saccharomyces cerevisiae OTCase is partially inhibited by L-ornithine at concentrations three times higher than the $K_{\mathrm{m}}$ value for this substrate (Messenguy \& Wiame, 1969). The site for inhibition is distinct from the catalytic site and can be lost by mutation, heating or chemical alteration, with the simultaneous loss of the ability to be inhibited by arginase. Hence partial inhibition of OTCase by ornithine has been proposed to be correlated with epiarginasic regulation (Messenguy et al., 1971). In the present work, we tested various yeast species to determine whether sensitivity to inhibition by arginase was always correlated with sensitivity to inhibition by ornithine at high concentrations (Table 2). The $K_{\mathrm{m}}$ values of different OTCases for ornithine varied from 0.6 to $17 \mathrm{mM}$, in most cases being from 1-5 mM, i.e. close to the value observed for Sacch. cerevisiae (3 mM). Inhibition by excess ornithine was highly variable. Some OTCases showed little or no inhibition, e.g. those of $H$. wingei, Deb. hansenii, Deb. phaffi, Deb. marama (Fig. $4 a$ ) and $H$. anomala. Inhibition comparable to Sacch. cerevisiae was common. More intense inhibition than Sacch. cerevisiae was unusual. There was no relationship between inhibition and $K_{\mathrm{m}}$ value. Saccharomyces cerevisiae, Deb. phaffii and T. candida had similar $K_{\mathrm{m}}$ values but their sensitivity to excess ornithine was intermediate, nil and high, respectively. However, correlation with sensitivity to arginase was absolute: all OTCases sensitive to arginase showed a marked inhibition by ornithine. The reverse was not true: some OTCases were insensitive to arginase but were inhibited by ornithine. This was the case for C. tropicalis, $T$. candida and Cr. neoformans 

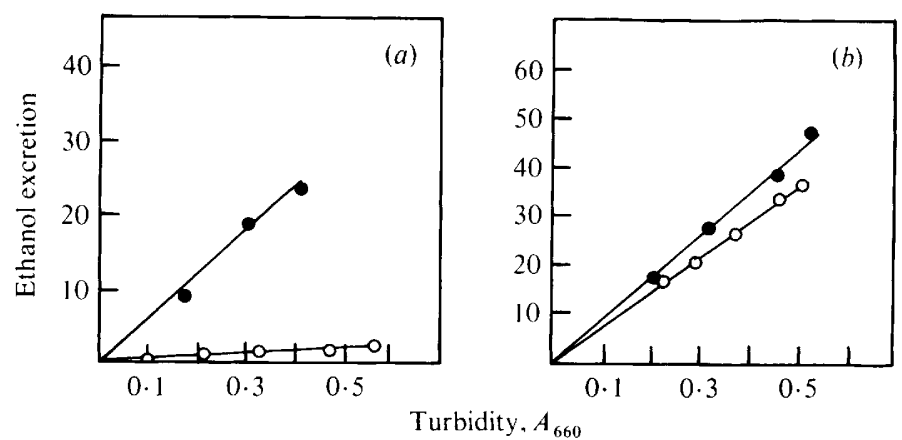

Fig. 5. Effect of aeration on the fermentation capacity of (a) Kluyveromyces fragilis and (b) Schizosaccharomyces pombe. Cells were grown aerobically $(O)$ and anaerobically $(\mathcal{O})$ in ammonium medium with $3 \%(\mathrm{w} / \mathrm{v})$ glucose, and the differential rate of ethanol excretion into the medium [expressed as $\mu \mathrm{mol}$ ( $\mathrm{ml}$ culture $)^{-1}$ ] was determined. Turbidity was measured with a Beckman B spectrophotometer.

(Fig. $4 b$ ), the last two being strongly inhibited by ornithine. Thus, it appears that inhibition by excess ornithine is necessary but not sufficient for the expression of epiarginasic regulation.

\section{Correlation with oxygen interaction}

The interaction of oxygen with yeasts is not limited to energy production. Severe anaerobiosis may impair growth in some fermentative yeasts. Some oxygen is required for lipid synthesis in Sacch. cerevisiae. Yeasts in which ethanol production is reduced by oxygen by more than $75 \%$ have been placed in the class of yeasts with a strong Pasteur effect. Kluyveromyces fragilis, $K$. lactis and Schiz. pombe grow well in usual anaerobic conditions but not in strict anaerobic conditions (Subik et al., 1974). As our purpose was to evaluate the reliability of the correlation of the origin of energy production and its regulation with epiarginasic regulation, we did not consider Kluyveromyces and similar species as obligate aerobes.

In Table 2, yeasts have been classified as obligate aerobes, facultative anaerobes with a strong Pasteur effect, and facultative anaerobes with a weak Pasteur effect. The Pasteur effect was measured by comparing ethanol production in yeasts growing either in a nitrogen-flushed atmosphere or in air (see Methods). We have confirmed the findings of Subik et al. (1974) concerning the strict anaerobic growth of Schiz. japonicus var. versatilis and the less strict growth patterns of Schiz. pombe and K. lactis. Table 2 shows that the need for a small amount of oxygen is not related to the Pasteur effect. The occurrence of the Pasteur effect reported in Table 2 confirms and extends previous results for Sacch. cerevisiae (Swanson \& Clifton, 1948; Lemoigne et al., 1954; Ephrussi et al., 1956) and other yeasts (Lafon, 1956; De Deken, 1966). Figure 5 illustrates the presence and the absence of a Pasteur effect.

Clearly the correlation between energy production from respiration and the absence of epiarginasic regulation is followed in many cases but is not complete. One needs to look critically at the exceptions as well as confirmed examples. Was the correlation only apparent and a result of a limited selection of yeasts? Would it be possible to rationalize the exceptions in relation to other properties? Among the obligate aerobes epiarginasic regulation was always absent. This seems well established, especially since we chose representative species from genera which also contained species capable of fermentation, such as Debaryomyces and Pichia. In addition, some of these epiarginasic negative yeasts had an OTCase strongly inhibited by ornithine, a property which appeared to be required for this regulation.

On the other hand, some fermentative yeasts which did not show the Pasteur effect did not show epiarginasic regulation. Brettanomyces had no Pasteur effect but its anaerobic growth was very slow and, in addition, this genus is known to have a peculiar metabolism. Oxygen is 


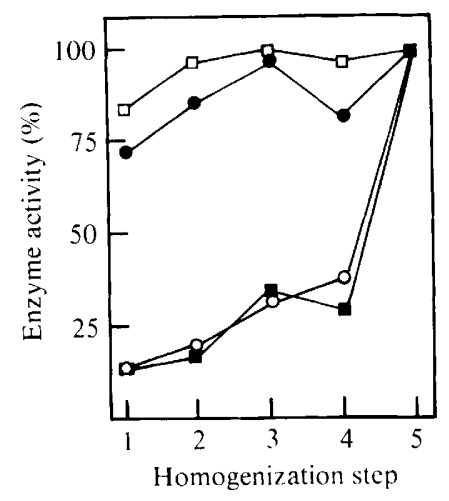

Fig. 6. Determination of the subcellular localization of OTCase and arginase in Schiz. japonicus var. versatilis by stepwise homogenization of spheroplasts. Enzyme activities were determined in the supernatant of each lysate obtained after $20 \mathrm{~min}$ centrifugation at $39000 \mathrm{~g}$. Each pellet corresponds to about $5 \mathrm{~g}$ wet wt cells. Enzymes: OTCase $(O)$; arginase $(\bigcirc)$; glucose-6-phosphate dehydrogenase $(\square)$; citrate synthase $(\boldsymbol{\square})$.

Step 1: the spheroplast pellet was suspended in Tris/ $\mathrm{HCl}$ buffer $(0.05 \mathrm{M}, \mathrm{pH} 7.4)$ containing $1 \mathrm{mM}-\mathrm{MgEDTA}, 0.1 \%$ bovine serum albumin and $1 \mathrm{M}$-mannitol (instead of $1.8 \mathrm{M}$ normally used for protection of spheroplasts). The suspension was homogenized by shaking the tube manually for $1 \mathrm{~min}$ and then using a Potter homogenizer for $5 \mathrm{~min}$.

Step 2: same treatment as in step 1 except that the mannitol concentration was $0.6 \mathrm{M}$.

Step 3: suspension in buffer containing $0.3 \mathrm{M}$-mannitol; manual homogenization for $2 \mathrm{~min}$, then the mannitol concentration was adjusted to $1 \mathrm{M}$ and homogenization was carried out with a Potter homogenizer for $2 \mathrm{~min}$.

Step 4: suspension in buffer only; 2 min manual homogenization and then the mannitol concentration was adjusted to $1 \mathrm{M}$.

Step 5: twofold passage of the spheroplast pellet through a French press $-100 \%$ activity corresponds to the activity recovered in this supernatant.

involved in some acid production (Custers, 1940). Thus, in this yeast oxygen affects the metabolism by a means other than the usual Pasteur effect.

A puzzling exception was Schiz. pombe. Although the Pasteur effect was absent, no epiarginasic regulation was found. This one instance could have been considered minor since Schiz. pombe needs at least some oxygen for growth. However, when we investigated other members of the group of fission yeasts, we found that all lacked the epiarginasic effect. Schizosaccharomyces japonicus was the most interesting example. Oxygen has adverse effects on its growth and sporulation (Wickerham \& Duprat, 1945) and cytochromes $b$ and $c$ are absent (Bulder, 1963). Its generation time in anaerobic conditions was $3.5 \mathrm{~h}$, whereas active oxygenation led to a generation time of $8 \mathrm{~h}$. In addition, its OTCase was found to be a mitochondrial enzyme (Fig. 6) when tested by the technique of stepwise lysis of spheroplasts (Ryan et al., 1973) previously applied to the study of other yeasts (Urrestarazu et al., 1977). Thus, we conclude that the Schizosaccharomyces group as a whole is a well established exception to the correlation between the absence of the Pasteur effect and epiarginasic regulation. It may be important in this connection that fission yeasts and budding yeasts have quite separate taxonomic relationships. One may propose that fission yeasts, which do not show epiarginasic regulation, retain OTCase in the mitochondria. In the case of Schiz. japonicus var. versatilis, many functions normally associated with mitochondria are absent but OTCase has been located in this organelle (Fig. 6). This last result establishes the independence of diverse mitochondrial functions.

In fermentative yeasts with a strong Pasteur effect, the presence of epiarginasic regulation was variable. However, here again, taxonomic relationships were obvious. Kluyveromyces species, which were found to be positive for epiarginasic regulation, are taxonomically related to the Saccharomyces, a strong positive genus. Other species in the group which showed a 
Table 3. Specific activity for OTCase and arginase in different yeast species after growth aerobically at $29^{\circ} \mathrm{C}$ with ammonia, ammonia plus arginine or arginine as nitrogen nutrient

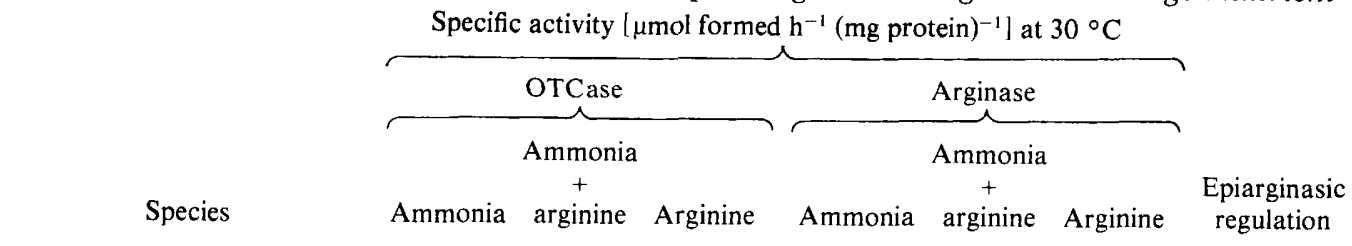

OBLIGATE AEROBES

Cr. neoformans
Deb. hansenii 767
Deb. hansenii 770
Deb. hansenii 792
Deb. hansenii 811
Deb. marama
H. wingei
P. fluxuum
T. candida

19
7
4
5
13
3
9
13
10

$\begin{array}{cc}16 & 13 \\ 3 & 2 \cdot 6 \\ 1 & 1 \\ 2 & 2 \cdot 7 \\ 3 & 2 \cdot 7 \\ 0.6 & 0 \cdot 6 \\ 13 & 11 \\ 2 & 0.5 \\ 2 & 1\end{array}$

9
7
3
51
3
3
15
8
12

$\begin{array}{rr}9 & 17 \\ 70 & 50 \\ 30 & 49 \\ 39 & 41 \\ 19 & 63 \\ 5 & 17 \\ 62 & 225 \\ 74 & 79 \\ 176 & 670\end{array}$

FACULTATIVE ANAEROBES WITH STRONG PASTEUR EFFECT

C. tropicalis

C. utilis

Deb. phaffii

H'spora valbyensis

H. anomala L-5-6

H. anomala diploid

K. fragilis

K. lactis

P.fermentans

P. dispora

9
13
2
14
9
7
30
25
6
13

$\begin{array}{ccr}0 \cdot 6 & 0 \cdot 5 & 2 \\ 6 & 7 & 1 \\ 1 & 0 \cdot 2 & 3 \\ 3 & 0 \cdot 3 & 3 \\ 10 & 11 & 12 \\ 8 & 8 & 10 \\ 12 & 2 & 8 \\ 10 & 4 & 6 \\ 1 \cdot 3 & 0 \cdot 8 & 6 \\ 20 & 14 & 13\end{array}$

2
1
3
3
12
10
8
6
6
13

$\begin{array}{rr}148 & 213 \\ 7 & 247 \\ 84 & 108 \\ 51 & 262 \\ 23 & 218 \\ 17 & 243 \\ 82 & 372 \\ 42 & 296 \\ 45 & 149 \\ 171 & 294\end{array}$

FACULTATIVE ANAEROBES WITH WEAK PASTEUR EFFECT

$\begin{array}{lrrrrrr}\text { Br. lambicus } & 35 & 12 & 1 & 0 \cdot 3 & 1 & 127 \\ \text { Schiz. japonicus var. jap. } & 18 & 4 & 3 & 67 & 269 & 465 \\ \text { Schiz. japonicus var. vers. } & 24 & 3 & 2 & 79 & 240 & 213 \\ \text { Schiz. pombe } & 9 & 6 & 6 & 33 & 92 & 379 \\ \text { Sacch. cerevisiae } & 30 & 2 & 1 & 6 & 20 & 291 \\ \text { Sacch. fermentati } 818 & 25 & 13 & 11 & 27 & 104 & 370 \\ \text { Sacch. kloeckerianus } & 19 & 15 & 7 & 10 & 25 & 270 \\ \text { S'codes ludwigii } & 22 & 10 & 6 & 4 & 39 & 240 \\ \text { T. colliculosa } & 28 & 1.5 & 1.5 & 12 & 198 & 290\end{array}$

strong Pasteur effect but no epiarginasic regulation belong to genera which include species that are obligate aerobes and are negative for this regulation.

\section{Regulation of arginase and OTCase synthesis}

As the purpose of epiarginasic regulation appears to be to avoid a futile cycle arising from the simultaneous presence of a catabolic enzyme (arginase) and an anabolic enzyme (OTCase), we have investigated the classical regulation of the synthesis of these enzymes. Results are reported in Table 3. Most yeasts showed the classical repression of OTCase and induction of arginase when arginine was added to minimal (ammonia) medium. In addition, they were subject to nitrogen catabolite repression, as judged by the increase in arginase synthesis when arginine was the only source of nitrogen compared with ammonia plus arginine as nitrogen nutrient. In this respect, the behaviour was similar to that of Sacch. cerevisiae (Béchet et al., 1970; Dubois et al., 1974). All epiarginasic positive yeasts showed strong arginase regulation.

In some yeasts, e.g. Cryptococcus, Debaryomyces, regulation was very poor; this was most common in obligate aerobes. From Table 3, it is clear that many species that lack the classical 
repression-induction mechanisms have not developed the epiarginasic regulation in compensation. On the contrary, poor regulation of synthesis was more common in obligate aerobes. In yeasts which lack classical regulation, compartmentation of OTCase could again be the basis for anabolism-catabolism exclusion.

The authors gratefully acknowledge the help of Professor M. Riley in the revision of the manuscript. This investigation was supported by grant no. 2.4549 .79 from the Fonds de la Recherche Fondamentale Collective (Belgium).

\section{REFERENCES}

BÉcheT, J, \& WiAME, J.-M. (1965). Indication of a specific regulatory binding protein for ornithine transcarbamylase in Saccharomyces cerevisiae. Biochemical and Biophysical Research Communications 21, 226-234.

Béchet, J., Grenson, M. \& Wiame, J.-M. (1970). Mutations affecting the repressibility of arginine biosynthetic enzymes in Saccharomyces cerevisiae. European Journal of Biochemistry 12, 31-39.

BonNichsen, R. (1963). In Methods of Enzymatic Analysis, pp. 285. Edited by H. U. Bergmeyer. New York: Academic Press.

Brewer, J. H. \& Allgeier, D. L. (1966). Safe self-contained carbon dioxide-hydrogen anaerobic system. Applied Microbiology 14, 985-988.

BuldER, C. J. E. A. (1963). On respiratory deficiency in yeasts. Dissertation, Delft, The Netherlands.

Custers, M. T. J. (1940). Onderzoekingen over het gistgeslacht Brettanomyces. Dissertation, Delft, The Netherlands.

De Deken, R. H. (1965). The Crabtree effect: a regulatory system in yeast. Journal of General Microbiology 44, 149-156.

Dubois, E., Grenson, M. \& Wiame, J.-M. (1974). The participation of the anabolic glutamate dehydrogenase in the nitrogen catabolite repression of arginase in Saccharomyces cerevisiae. European Journal of Biochemistry 48, 603-616.

Ephrussi, B., Slonimski, P. P., Yotsuyanagi, Y. \& TAVLITZKI, J. (1956). Variations physiologiques et cytologiques de la levure au cours du cycle de la croissance aérobie. Comptes rendus des travaux du Laboratoire Carlsberg (Série Physiologique) 26, 87-102.

Gamble, J. G. \& Lehninger, A. L. (1973). Transport of ornithine and citrulline across the mitochondrial membrane. Journal of Biological Chemistry 248, 610-618.

Grenson, M., Mousset, M., Wiame, J.-M. \& BÉCHET, J. (1966). Multiplicity of the amino acid permeases in Saccharomyces cerevisiae. Biochimica et biophysica acta 127, 325-338.

Issaly, I. M. \& Issaly, A. S. (1974). Control of ornithine carbamoyltransferase by arginase in Bacillus subtilis. European Journal of Biochemistry 49, 485-495.

LAFON, M. (1956). Sur quelques caractères physiologiques et biochimiques des levures de vin. Annales de l'Institut Pasteur 91, 91-99.

Lemoigne, M., Aubert, J. P. \& Millet, J. (1954). La production d'alcool et le rendement de croissance de la levure de boulangerie cultivée en aérobiose. Annales de l'Institut Pasteur 87, 427-439.

LodDer, J. (1970). The Yeasts. A Taxonomic Study, 2nd edn. Amsterdam: North Holland Publishing Co.

Messenguy, F. \& Wiame, J.-M. (1969). The control of ornithine transcarbamylase activity by arginase in Saccharomyces cerevisiae. FEBS Letters 3, 47-49.

Messenguy, F., Penninckx, M. \& Wiame, J.-M. (1971). Interaction between arginase and ornithine carbamoyltransferase in Saccharomyces cerevisiae. European Journal of Biochemistry 22, 277-286.

Monod, J., Pappenheimer, A. M., JR \& CohenBAZIRE, G. (1952). La cinétique de la biosynthèse de la $\beta$-galactosidase chez $E$. coli considérée comme fonction de la croissance. Biochimica et biophysica acta 9, 648-660.

PENNINCKX, M. (1975). Interaction between arginase and L-ornithine carbamoyltransferase in Saccharomyces cerevisiae. European Journal of Biochemistry 58, 533-538.

Penninckx, M. \& Wiame, J.-M. (1976). Affinity of arginase for ornithine carbamoyltransferase in Saccharomyces cerevisiae. Journal of Molecular Biology 104, 819-831.

Penninckx, M., Simon, J.-P. \& Wiame, J.-M. (1974). Interaction between arginase and L-ornithine carbamoyltransferase in Saccharomyces cerevisiae. Purification of Saccharomyces cerevisiae enzymes and evidence that these enzymes as well as rat liver arginase are trimers. European Journal of Biochemistry 49, 429-442.

Price, C. W., Fuson, G. B. \& Phaff, H. J. (1978). Genome comparison in yeast systematics: delimitation of species within the genera Schwanniomyces, Saccharomyces, Debaryomyces and Pichia. Microbiological Reviews 42, 161-193.

Ramos, F. \& Wiame, J.-M. (1979). Synthesis and activation of asparagine in asparagine auxotrophs of Saccharomyces cerevisiae. European Journal of Biochemistry 94, 409-417.

Roon, R. J., HAMpSHIRe, J. \& LEVENBERG, B. (1972). Urea amidolyase: the involvement of biotin in urea cleavage. Journal of Biological Chemistry 247, 7539-7545.

Ryan, E. D., Tracy, J. W. \& Kohlhaw, G. B. (1973). Subcellular localization of leucine biosynthetic enzymes in yeast. Journal of Bacteriology 116, 222-225.

RYTKA, J. (1975). Positive selection of general amino acid permease mutants in Saccharomyces cerevisiae. Journal of Bacteriology 121, 562-570. 
Subik, J., Kolarov, J. \& Kovac, L. (1974). Anaerobic growth and formation of respirationdeficient mutants of various species of yeasts. $F E B S$ Letters 45, 263-266.

Swanson, W. H. \& Clifton, C. E. (1948). Growth and assimilation in cultures of Saccharomyces cerevisiae. Journal of Bacteriology 56, 115-124.

Urrestarazu, L. A., Vissers, S. \& Wiame, J.-M. (1977). Change in location of ornithine carbamoyltransferase and carbamoylphosphate synthetase among yeasts in relation to the arginase/ornithine carbamoyltransferase regulatory complex and the energy status of the cells. European Journal of Biochemistry 79, 473-481.

Weiss, R. L. \& Davis, R. H. (1973). Intracellular localization of enzymes of arginine metabolism in Neurospora. Journal of Biological Chemistry 248. 5403-5408.
Wiame, J.-M. (1971). The regulation of arginine metabolism in Saccharomyces cerevisiae: exclusion mechanisms. In Current Topics in Cellular Regulation vol. 4, pp. 1-38. Edited by B. L. Horecker \& E. R. Stadtman. New York \& London: Academic Press.

Wiame, J.-M. (1975). Evolving of arginase, ornithine carbamoyltransferase interaction. In Mechanisms of Action and Regulation of Enzymes, Proceedings of the Ninth Meeting of the European Biochemical Society, Budapest, 1974, vol. 34, pp. 161-177. Edited by T. Keleti. New York: North-Holland/ American Elsevier.

Wickerham, L. J. \& Duprat, E. (1945). A remarkable fission yeast, Schizosaccharomyces versatilis nov.sp. Journal of Bacteriology, 50, 597-607. 